\title{
Quantum chaos in a schematic shell model
}

\author{
D. C. Meredith, ${ }^{*}$ S. E. Koonin, and M. R. Zirnbauer ${ }^{\dagger}$ \\ W. K. Kellogg Radiation Laboratory, California Institute of Technology, Pasadena, California 91125
}

(Received 26 October 1987)

\begin{abstract}
To test the connection between chaotic classical motion and quantum spectral and overlap statistics, we examine a schematic three-orbital shell model. This system is novel in that the quantum phase space is compact and the momentum dependence of the classical Hamiltonian is nonstandard. We find good agreement with the expected behavior of the spectral statistics and reasonable agreement for the overlap distributions. Also, there is evidence that the eigenvector statistics are more sensitive to the details of the classical dynamics than are the eigenvalues.
\end{abstract}

\section{INTRODUCTION}

The presence of chaos (i.e., sensitive dependence on initial conditions) in a classical system must influence the corresponding quantum system. ${ }^{1}$ There is much current effort to decide what quantitative properties of quantum systems clearly and unambiguously reflect the character of the classical dynamics. ${ }^{2,3}$ One approach, which we will follow, is to examine the spectral statistics for evidence of classical chaos.

The spectral statistics of a large class of quantum systems can be described in terms of random matrix theory (RMT). ${ }^{4,5}$ For example, experimental data from neutron resonance states in nuclei, ${ }^{6}$ bound states in atoms, and molecular vibronic energy states $^{3}$ all display nearestneighbor spacing distributions characteristic of the Gaussian orthogonal ensemble (GOE). Numerically generated spectra of quantum billiards (a particle in a twodimensional box) have GOE statistics if the classical ana$\log$ is chaotic and have Poisson statistics if it is integrable (i.e., if there are two conserved quantities). ${ }^{7,8}$ A system of two interacting particles in one dimension has statistics that vary smoothly from GOE to Poisson as the classical motion varies from chaotic to integrable. ${ }^{9}$

The pattern that has become apparent from these physical and numerical experiments is that complicated and chaotic systems have GOE statistics, while integrable systems have Poisson statistics, and systems with intermediate dynamics have intermediate statistics. There are semiclassical arguments that support this hypothesis and also display some new features that go beyond GOE. ${ }^{10-12}$

The present study is a novel test of this hypothesis. The three-orbital shell model differs from previously studied systems because the quantum basis states are finite in number, the classical phase space is compact, and the momentum dependence of the classical Hamiltonian is not of the conventional form. It is also clearly closer in structure to the nuclear systems that evidence GOE behavior than are the "particle-in-a-box" systems.

Our discussion begins with a description of the model. The main body of the paper includes numerical calculation of the eigenvalues and eigenvectors of the quantum system, derivation of the classical limit, determination of the classical dynamics, and finally an analysis of the spectral fluctuations and eigenvector projections on a complete set of basis states.

\section{QUANTUM MODEL}

The model that we study is the three-orbital LipkinMeshkov-Glick (LMG) model, ${ }^{13}$ which was introduced in an effort to check the validity of approximate many-body techniques, including the random-phase approximation and Bardeen-Cooper-Schrieffer (BCS) theory. This is a nontrivial model, analytically soluble in a few simple cases and numerically soluble in others, which mimics the shell-model picture of the nucleus.

The model has $M$ particles that are distributed among three energy orbitals, each of which is $\boldsymbol{M}$-fold degenerate. The single-particle states are labeled by two numbers, $k=0,1,2$ for the ground, first, and second excited orbitals, and $m=1,2,3, \ldots, M$ for the degenerate states within each orbital. The energy for each orbital is denoted by $\varepsilon_{k}$.

Using the language of second quantization, the Hamiltonian is

$$
\begin{aligned}
H= & \sum_{k=0}^{2} \varepsilon_{k}\left[\sum_{m=1}^{M} a_{k m}^{\dagger} a_{k m}\right] \\
& -\frac{1}{2} \sum_{k, l=0}^{2} V_{k l}\left[\sum_{m=1}^{M} a_{k m}^{\dagger} a_{l m}\right]^{2},
\end{aligned}
$$

where $a^{\dagger}$ and $a$ are fermionic and creation and annihilation operators that obey the usual anticommutation relations. In all of our calculations we place the orbitals symmetrically about zero, $\varepsilon_{2}=-\varepsilon_{0} \equiv \varepsilon, \varepsilon_{1}=0$. We also choose vanishing interactions for particles in the same orbital and equal interactions for particles in different orbitals [i.e., $V_{k l} \equiv V\left(1-\delta_{k l}\right)$ ]. All our energies are quoted in units of $M \varepsilon$, and we define the dimensionless coupling constant $\chi \equiv M V / \varepsilon$.

Because there is only a finite number of ways of putting $M$ particles in three orbitals, there is a finite number of basis states for the LMG model. This makes the quantum calculations straightforward, as basis truncation errors are eliminated, but it also makes the LMG model 
qualitatively different from those studied previously. In addition, there are two symmetries that decrease the numerical burden for a given $\boldsymbol{M}$.

To see the first symmetry, we define the nine twofermion operators that are symmetric under interchange of the particle labels,

$$
G_{k l} \equiv \sum_{m=1}^{M} a_{k m}^{\dagger} a_{l m} .
$$

The Hamiltonian can be written in terms of these operators only,

$$
H=\sum_{k=0}^{2} \varepsilon_{k} G_{k k}-\frac{1}{2} \sum_{k, l=0}^{2} V\left(1-\delta_{k l}\right) G_{k l}^{2},
$$

and thus conserves permutation symmetry of the particle labels. In particular, the Hamiltonian couples symmetric states only with other symmetric states. It is this subspace that we use in our quantum calculations. The dimension of this symmetric basis is $\mathcal{N}=(M+2)(M$ $+1) / 2$, the number of ways of putting $M$ particles in three orbitals when order does not matter. The states of other permutation symmetry, which we did not consider, should have qualitatively the same behavior, but they are systems with more degrees of freedom that do not approach the classical limit as rapidly as do the totally symmetric states.

The second symmetry arises because the interaction moves only pairs of particles. Therefore $H$ conserves the oddness or evenness of the population in each orbital (which we refer to as the signature). There are thus four different matrices for each value of $M$, identified by $s_{0} s_{1} s_{2}$, the signature of each orbital. For $M$ even these matrices are referred to as eee, eoo, ooe, and oeo; for $M$ odd, the possibilities are ooo, oee, eeo, and eoe. After all the symmetries are exploited, the final dimension of each matrix that must be diagonalized numerically is $\mathcal{N} \approx M^{2} / 8$.

The eigenstates of the number operator for each orbital are a convenient basis for the quantum calculations. They are labeled by $(b, c)$, where $b$ is the number of particles in the first excited orbital and $c$ the number in the second. By conservation of particle number, the population of the ground orbital is $a=M-b-c$. The noninteracting ground state has all $\boldsymbol{M}$ particles in the ground orbital, and is represented by $|00\rangle$. The other basis states are written using the symmetric raising operators

$$
|b c\rangle=C(b, c) G_{10}^{b} G_{20}^{c}|00\rangle,
$$

where $C(b, c)$ is the normalizing coefficient. Using the commutation relation for these operators,

$$
\left[G_{k l}, G_{k^{\prime} l^{\prime}}\right]=G_{k l^{\prime}} \delta_{k^{\prime} l}-G_{k^{\prime} l} \delta_{k l^{\prime}},
$$

we can calculate the matrix elements ${ }^{14}$

$$
\begin{aligned}
\frac{H_{b^{\prime} c^{\prime}, b c}}{M \varepsilon} & \equiv\left\langle b^{\prime} c^{\prime}\left|\frac{H}{M \varepsilon}\right| b c\right\rangle \\
& =\frac{1}{M}(-M+b+2 c) \delta_{b b^{\prime}} \delta_{c c^{\prime}}-\frac{\chi}{2 M^{2}} A_{b^{\prime} c^{\prime}, b c},
\end{aligned}
$$

where

$$
\begin{aligned}
A_{b^{\prime} c^{\prime}, b c}= & \sqrt{b(b-1)(M-b-c+1)(M-b-c+2)} \delta_{b-2, b^{\prime}} \delta_{c c^{\prime}} \\
& +\sqrt{(b+1)(b+2)(M-b-c)(M-b-c-1)} \delta_{b+2, b^{\prime}} \delta_{c c^{\prime}} \\
& +\sqrt{c(c-1)(M-b-c+1)(M-b-c+2)} \delta_{b b^{\prime}} \delta_{c-2, c^{\prime}}+\sqrt{(c+1)(c+2)(M-b-c)(M-b-c-1)} \delta_{b b^{\prime}} \delta_{c+2, c^{\prime}} \\
& +\sqrt{(b+1)(b+2) c(c-1)} \delta_{b+2, b^{\prime}} \delta_{c-2, c^{\prime}}+\sqrt{(c+1)(c+2) b(b-1)} \delta_{b-2, b^{\prime}} \delta_{c+2, c^{\prime}} .
\end{aligned}
$$

The Kronecker symbols show explicitly preservation of signature.

Each matrix is sparse (there are no more than seven nonzero elements in each row) and banded (since $\left|b-b^{\prime}\right| \leq 2$ and $\left.\left|c-c^{\prime}\right| \leq 2\right)$. We used the IMSL Fortran software library diagonalization routine EIGBS, tailored for banded, real, symmetric matrices. This employs the Householder algorithm to reduce the matrix to tridiagonal form, and then calls an iterative routine (known in the literature as the $\mathrm{QR}$ algorithm) to find the eigenvalues and eigenvectors. ${ }^{15}$ As a check on the packaged routine, we calculated the trace of $H$ and $H^{2}$ before and after diagonalization; these were conserved to at least one part in $10^{8}$.

Eigenvalue calculations were done for several different values of $\boldsymbol{M}$. The largest matrix we diagonalized was for $M=120$, giving $\mathcal{N}=1830$ or 1891 . Eigenvectors were done only for $M=85$. A measure of quality for the eigenvectors is given by

$$
\max _{1 \leq j \leq \mathcal{N}} \frac{\left|H \Psi_{j}-E_{j} \Psi_{j}\right|}{\sqrt{\operatorname{Tr}\left(H^{2}\right)}},
$$

where the numerical eigenvalues and eigenvectors are denoted by $E_{j}$ and $\Psi_{j}$. The largest value for this quantity was $3 \times 10^{-8}$.

\section{CLASSICAL DYNAMICS}

\section{A. Classical limit}

To test the hypothesis relating level fluctuations to classical dynamics, we must, of course, study the latter, and in particular determine for what values of the coupling strength the trajectories are chaotic or integrable. This, in turn, requires that we derive the classical limit of the LMG model.

We begin this derivation by introducing coherent 
states, which are written in terms of the symmetric raising operators, ${ }^{16}$

$$
\left|\Phi\left(z_{1}, z_{2}\right)\right\rangle \equiv \exp \left(z_{1} G_{10}+z_{2} G_{20}\right)|00\rangle,
$$

where $z_{1}$ and $z_{2}$ are complex numbers that might depend on time.

These coherent states are a natural choice for describing classical behavior. ${ }^{17}$ This is because, for $M \rightarrow \infty$, all the quantum effects disappear: matrix elements of operators between different coherent states are zero (therefore states do not interfere), expectation values of operators factorize,

$$
\langle A B\rangle \rightarrow\langle A\rangle\langle B\rangle+O\left(\frac{1}{M}\right),
$$

giving (with $A=B$ ) zero uncertainty in the value for any operator, and a time-evolved coherent state (which is not itself a coherent state) spreads at a vanishing small rate proportional to $M^{-1}$.

The normalization of these coherent states is given by ${ }^{16}$

$$
\langle\Phi(z) \mid \Phi(z)\rangle=\left(1+\left|z_{1}\right|^{2}+\left|z_{2}\right|^{2}\right)^{M},
$$

and they obey the completeness relation

$$
\begin{aligned}
\int(M+1)(M+2) & \frac{|\Phi(z)\rangle\langle\Phi(z)|}{(2 \pi)^{2}} \\
& \times \frac{d z_{1} d z_{1}^{*} d z_{2} d z_{2}^{*}}{\left(1+\left|z_{1}\right|^{2}+\left|z_{2}\right|^{2}\right)^{M+3}}=1 .
\end{aligned}
$$

Because the wave functions are not normalized and the integration measure is not unity, the $z$ 's are not the most convenient parametrization of the coherent states. Instead, we work with the normalized states

$$
|\Psi(z)\rangle \equiv \frac{|\Phi(z)\rangle}{\sqrt{\langle\Phi(z) \mid \Phi(z)\rangle}},
$$

and change to the variables

$$
\beta_{j} \equiv \frac{z_{j}}{\left(1+\left|z_{1}\right|^{2}+\left|z_{2}\right|^{2}\right)^{1 / 2}}, \quad j=1,2 .
$$

The completeness relation now reads

$$
\int(M+1)(M+2) \frac{|\Psi(\beta)\rangle\langle\Psi(\beta)|}{(2 \pi)^{2}} d \beta_{1} d \beta_{1}^{*} d \beta_{2} d \beta_{2}^{*}=1 \text {. }
$$

Time evolution is affected by the propagator

$$
\left|\Psi\left(t_{f}\right)\right\rangle=e^{-i H\left(t_{f}-t_{i}\right)}\left|\Psi\left(\beta_{i}\right)\right\rangle .
$$

The overlap between an arbitrary final coherent state and our time-evolved initial state is given by

$$
\left\langle\Psi\left(\beta_{f}\right)\left|e^{-i H\left(t_{f}-t_{i}\right)}\right| \Psi\left(\beta_{i}\right)\right\rangle,
$$

where $\beta_{i}$ and $\beta_{f}$ denote two different sets of $\beta$ values. To evaluate this integral, we follow the standard pathintegral approach ${ }^{16,18}$ by rewriting the exponential as a product of $L$ exponentials and then inserting unity, as expressed by Eq. (15) between two successive terms in the product. In the limit $L \rightarrow \infty$, the overlap in Eq. (17) can be written as

$$
\int_{\beta_{0}}^{\beta_{L}} \mathcal{D}(\beta) e^{i M S(\beta)},
$$

where $\mathcal{D}(\beta)$ indicates an integration over all paths $\beta(t)$, and the action $S$ is defined by

$$
S(\beta) \equiv \frac{1}{M} \int_{t_{i}}^{t_{f}}\left\langle\Psi(\beta(t))\left|i \frac{\partial}{\partial t}-H\right| \Psi(\beta(t))\right\rangle .
$$

It can be shown that this action is independent of $M$.

Because the phase in the path integral is proportional to $M$, we are justified in using the stationary-phase approximation to evaluate the integral in the limit $M \rightarrow \infty$. Thus we see that $M$ plays the role of $\hbar^{-1}$ in conventional quantum mechanics. In this limit, the only paths that contribute satisfy the variational condition $\delta S=0$. This gives

$$
\begin{aligned}
\frac{\partial\langle H / M\rangle}{\partial \beta_{k}}=\frac{i}{M} \sum_{j=1,2}\left[\dot{\beta}_{j}\right. & \frac{\partial}{\partial \beta_{j}}\left\langle\Psi \mid \Psi_{\beta_{k}}\right\rangle \\
& \left.-\frac{\partial}{\partial \beta_{k}}\left\langle\Psi \mid \Psi_{\beta_{j}}\right\rangle\right] \\
+\dot{\beta}_{j}^{*} & {\left[\frac{\partial}{\partial \beta_{j}^{*}}\left\langle\Psi \mid \Psi_{\beta_{k}}\right\rangle\right.} \\
& \left.\left.-\frac{\partial}{\partial \beta_{k}}\left\langle\Psi \mid \Psi_{\beta_{j}^{*}}\right\rangle\right]\right],
\end{aligned}
$$

where

$$
\left|\Psi_{\beta_{j}}\right\rangle \equiv \frac{\partial}{\partial \beta_{j}}|\Psi\rangle
$$

and the $\beta$ dependence in $\Psi$ has been suppressed.

$\mathrm{Kan}^{19}$ gives a general method for finding new variables to bring these equations of motion for the parameters into canonical form. However, the $\beta_{j}$ 's are in fact the correct variables. To see this, note that

$$
\left\langle\Psi \mid \Psi_{\beta_{j}}\right\rangle=\frac{M}{2} \beta_{j}^{*}, \quad\left\langle\Psi \mid \Psi_{\beta_{j}^{*}}\right\rangle=-\frac{M}{2} \beta_{j} .
$$

Using these relations in Eq. (20) we obtain

$$
\frac{\partial\langle H / M\rangle}{\partial \beta_{j}}=i \dot{\beta}_{j}^{*}, \quad \frac{\partial\langle H / M\rangle}{\partial \beta_{j}^{*}}=-i \dot{\beta}_{j} .
$$

We eliminate the factor $i$ by one last change of variables,

$$
\beta_{j}=\frac{q_{j}+i p_{j}}{\sqrt{2}}, \quad \beta_{j}^{*}=\frac{q_{j}-i p_{j}}{\sqrt{2}},
$$

and identify $p$ and $q$ as canonical momentum and position variables for $H_{\text {class }} \equiv\langle\Psi|H / M| \Psi\rangle$.

In terms of the canonical variables,

$$
\begin{aligned}
\frac{H_{\text {class }}(\mathbf{q}, \mathbf{p})}{\varepsilon}= & -1+\frac{1}{2} q_{1}^{2}(1-\chi)+\frac{1}{2} q_{2}^{2}(2-\chi) \\
& +\frac{1}{2} p_{1}^{2}(1+\chi)+\frac{1}{2} p_{2}^{2}(2+\chi) \\
& +\frac{1}{4} \chi\left[\left(q_{1}^{2}+q_{2}^{2}\right)^{2}-\left(p_{1}^{2}+p_{2}^{2}\right)^{2}\right. \\
& \left.\quad-\left(q_{1}^{2}-p_{1}^{2}\right)\left(q_{2}^{2}-p_{2}^{2}\right)-4 q_{1} q_{2} p_{1} p_{2}\right] .
\end{aligned}
$$


Note the appearance of terms that are fourth order in $p$; this Hamiltonian is clearly not that of a particle in a potential well.

The only adjustable parameter is $\chi \equiv M V / \varepsilon$, the normalized strength of the two-particle interaction. As $\chi$ varies, the topology of the "potential" surface, defined by $H(\mathrm{q}, \mathrm{p}=0)$, changes. Because of the quartic momentum dependence of $H$, this surface does not have the standard interpretation, but it is still informative (Fig. 1). The number and location of the minima vary with $\chi$ as shown in Table I. The energy maxima also vary with $\chi$ : for $\chi=0, E_{\max }=1$ (all particles in the highest orbital), and for $\chi \rightarrow \infty$ numerical calculations give $E_{\max } \approx 0.255 \chi$.

The canonical variables $(q, p)$ can be expressed in terms of expectation values of the symmetric operators,

$p_{j}=-i \frac{\left\langle G_{0 j}\right\rangle-\left\langle G_{j 0}\right\rangle}{\sqrt{2 M\left\langle G_{00}\right\rangle}}, \quad q_{j}=\frac{\left\langle G_{0 j}\right\rangle+\left\langle G_{j 0}\right\rangle}{\sqrt{2 M\left\langle G_{00}\right\rangle}}$.

Twice the fraction of particles in the $j$ th orbital $(j=1,2)$ is given by

$$
2 \frac{\left\langle G_{j j}\right\rangle}{M}=p_{j}^{2}+q_{j}^{2} .
$$

Since the number of particles is conserved, we must have

$$
r^{2} \equiv p_{1}^{2}+q_{1}^{2}+p_{2}^{2}+q_{2}^{2} \leq 2 \text {. }
$$

The phase space for the classical Hamiltonian is thus compact. Indeed, examining the equation of motion for $r$ with $\delta \equiv 2-r^{2}$ small, we find $\dot{r} \propto \delta$; a trajectory can never cross the surface at $r^{2}=2$.

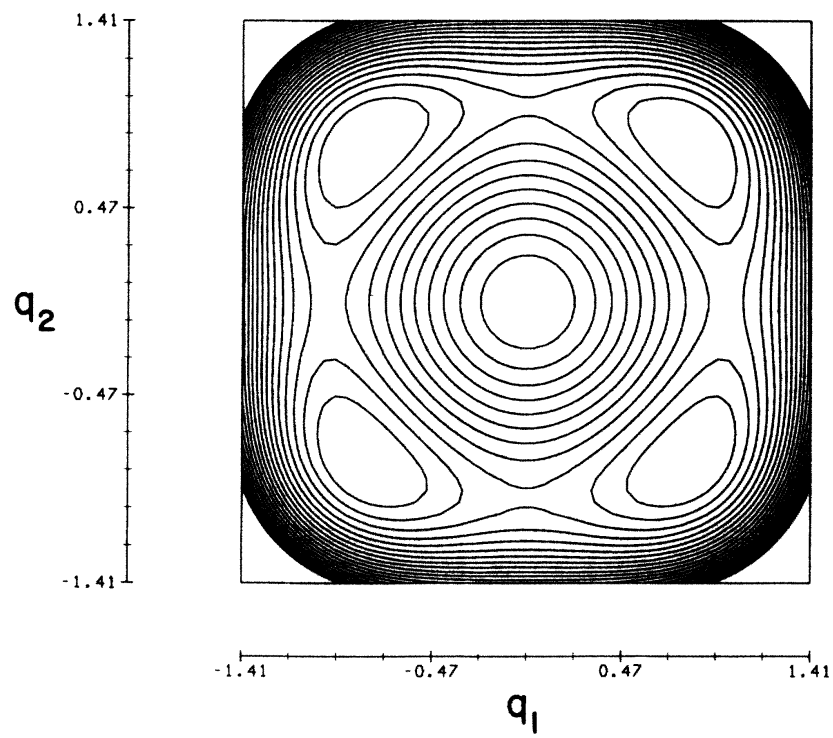

FIG. 1. Potential energy surface $[H(\mathbf{q}, \mathbf{p}=0)]$ for the classical LMG model [Eq. (25)] with $\chi=100$. There are four minima at $\left(q_{1}^{2}=\frac{2}{3}, q_{2}^{2}=0.65\right)$ with $E_{\mathrm{min}}=-33.34$, a local maximum at $\left(q_{1}=0, q_{2}=0\right)$ with $E=-1$, and four saddle points at $\left(q_{1}^{2}=0.99, q_{2}=0.0\right)$ with $E=-25.5$ and at $\left(q_{1}=0.0, q_{2}^{2}=0.98\right)$ with $E=-25.01$.
TABLE I. Locations $\left(q_{10}, q_{20}\right)$ and values $\left(E_{\min }\right)$ of the minima of the potential energy surface [Eq. (25)] as a function of the normalized coupling constant $\chi$.

\begin{tabular}{cccc}
\hline \hline & $q_{10}^{2}$ & $q_{20}^{2}$ & $E_{\min }$ \\
\hline$\chi \leq 1$ & 0 & 0 & -1 \\
$1 \leq \chi \leq 3$ & $1-\frac{1}{\chi}$ & 0 & $-1-\frac{(\chi-1)^{2}}{4 \chi}$ \\
$\chi \geq 3$ & $\frac{2}{3}$ & $\frac{2 \chi-6}{3 \chi}$ & $-\frac{\chi}{3}-\frac{1}{\chi}$ \\
\hline
\end{tabular}

\section{B. Classical chaos}

The next step involves determining the character of the classical dynamics of the LMG model. Before doing so, we review some aspects of the theory of classical nonlinear dynamics.

A classical system is chaotic (more technically, a $K$ system, so named to honor Kolmogorov) if it is ergodic (almost every trajectory passes arbitrarily close to almost every point in phase space) and if the phase-space trajectories are linearly unstable. ${ }^{20}$ The stability of a trajectory is measured by the Lyapunov exponent, which is defined as follows. Let $\mathrm{x}$ stand for all $2 N$ phase-space variables and write the equations of motion as

$$
\dot{\mathbf{x}}=\mathbf{F}(\mathbf{x}) \text {. }
$$

Each trajectory is denoted by $\mathbf{x}\left(\mathbf{x}_{0}, t\right)$, explicitly showing the dependence on initial conditions and time. Now, define a vector $\boldsymbol{\eta}$ between two initially infinitesimally close trajectories, ${ }^{21}$

$$
\boldsymbol{\eta}\left(\mathbf{x}_{0}, t, \hat{\boldsymbol{\delta}}\right) \equiv \lim _{|\boldsymbol{\delta}| \rightarrow 0} \frac{\mathbf{x}\left(\mathbf{x}_{0}+\boldsymbol{\delta}, t\right)-\mathbf{x}\left(\mathbf{x}_{0}, t\right)}{|\boldsymbol{\delta}|} .
$$

The time evolution for $\boldsymbol{\eta}$ is given by the linearized equations of motion,

$$
\dot{\boldsymbol{\eta}}\left(\mathbf{x}_{0}, t\right)=\underline{D}^{F} \boldsymbol{\eta}\left(\mathbf{x}_{0}, t\right),
$$

where

$$
\left.D_{i j}^{F} \equiv \frac{\partial F_{i}}{\partial x_{j}}\right|_{\mathbf{x}\left(\mathbf{x}_{0}, t\right)} .
$$

The Lyapunov exponent is then defined by

$$
\lambda\left(\mathbf{x}_{0}, \hat{\boldsymbol{\delta}}\right) \equiv \lim _{t \rightarrow \infty} \frac{1}{t} \ln \frac{\left|\boldsymbol{\eta}\left(\mathbf{x}_{0}, t, \hat{\boldsymbol{\delta}}\right)\right|}{\left|\boldsymbol{\eta}\left(\mathbf{x}_{0}, 0, \hat{\boldsymbol{\delta}}\right)\right|} .
$$

This exponent measures the time-averaged rate of exponential separation. A positive exponent indicates that the trajectory is linearly unstable, while a negative exponent indicates that it is stable. It can be shown that this exponent, in the infinite-time limit, is independent of $\hat{\boldsymbol{\delta}}$ for almost every choice of $\hat{\boldsymbol{\delta}}{ }^{21}$

But chaos is only one possible type of classical dynamics: the system could also be integrable, or lie somewhere between these two extremes. For an integrable system with two degrees of freedom, there is a second conserved quantity (in addition to the energy), which forces trajec- 
tories to lie on a two-dimensional torus in phase space. For systems that are near integrable, the KolmogorovArnold-Moser (KAM) theorem proves that most tori persist, although distorted. ${ }^{22}$ In a system with two degrees of freedom, these KAM tori partition phase space and prevent the ergodicity necessary for a $K$ system.

Returning to the LMG model, note that for $\chi=0$ [see Eq. (25)] the system is a two-dimensional oscillator and therefore integrable. As $\chi$ increases, the system might vary from integrable to chaotic. Also, for a fixed value of $\chi$, varying the energy might change the nature of the dynamics: near $E_{\text {min }}$ (see Table I) the motion is smallamplitude oscillations about the minima and therefore quasi-integrable, but for higher energies this might not be true. Therefore, a thorough search for chaos should be done in the energy- $\chi$ plane. In practice, we looked at a few values of $\chi$ and were satisfied with numerical evidence for ergodicity and chaos.

We chose four values of $\chi$ for the initial search: 0.75 , 2,10 , and 100 corresponding to one, two, four, and again four minima in the potential surface. We did not calculate Lyapunov exponents at first, but instead looked at surfaces of section. ${ }^{20}$ For a system with two degrees of freedom, a surface of section is the intersection of a trajectory with a plane in the three-dimensional phase space (the fourth variable is fixed by energy conservation), i.e., a set of points. If the system is a $K$ system (and therefore ergodic), the surface of section is a set of points evenly covering all of the energetically available phase space, with no KAM tori. If the system is integrable, the tori are seen as closed curves in the surface of section. Systems that lie between chaotic and integrable have surfaces of section with both closed curves and with clusters of points covering some proper subset of the available phase space.

The surfaces of section were done for 10 to 15 energies from the allowed energy range for each value of $\chi$. The equations of motion were integrated numerically using a fourth-order Runge-Kutta algorithm with a step size chosen to conserve energy to at least one part in $10^{3}$. Making a $\left(p_{2}, q_{2}\right)$ surface of section, we triggered on the crossings of the $q_{1}=q_{10}$ plane, the equilibrium value of $q_{1}$ (Table I), and also required that $\dot{q}_{1} \geq 0$. Because the momentum dependence of the energy is quartic, there can be two values of $p_{1}$ in the allowed range [Eq. (28)] that satisfy the trigger conditions and conserve energy. Therefore, to uniquely specify $p_{1}$, the trigger for the surface of section also required that $p_{1}$ be the larger of the two allowed values. When the trigger fired, we integrated the equations of motion exactly back to the surface by changing the independent variable to $q_{1}$ and the step length to $q_{10}-q_{1}$. This numerical method of finding surface of section points is due to Henon. ${ }^{23}$

For $\chi=0.75$ nothing but KAM tori appear at all energies; the system is close to integrable. For $\chi=2$ and 10 there are KAM tori at all energies as well as chaotic regions in the middle of the spectrum. For $\chi=100$ (Fig. 2) there is a large energy range (about -25 to 0 ) for which the motion is apparently totally chaotic. We need a long energy range with completely chaotic dynamics so that we have enough eigenvalues in the quantum system for
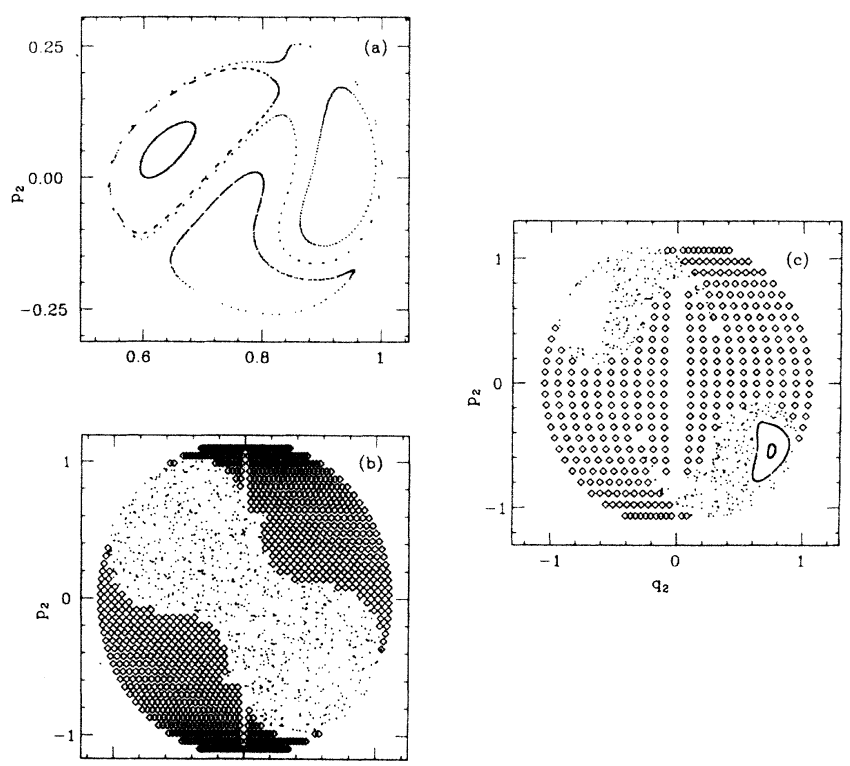

FIG. 2. $\left(q_{2}, p_{2}\right)$ surfaces of section for (a) $E=-30$, (b) $E=-2$, and (c) $E=10$ for the LMG model, with $\chi=100$ and $q_{1}=q_{10}=0.8165$. The diamonds in (b) and (c) are centered on points that are energetically inaccessible. In panel (a) there are five closed curves from five sets of initial conditions; their presence indicates that the dynamics are nearly integrable. Panel (b) shows a sea of points that were generated from one trajectory and that cover the entire available surface; this indicates that the system is ergodic. Panel (c) shows features of the other two, i.e., closed curves and clusters of dots, confirming that the classical dynamics lie between chaotic and integrable.

meaningful statistical analysis. For this reason, further investigations were limited to $\chi=100$.

Although the surfaces of section give a good qualitative picture of the topology of phase space, we needed a more quantitative test for complete chaos. If a system is ergodic, then the calculation of the Lyapunov exponent tells all: a positive Lyapunov exponent proves the system is a $K$ system while a zero exponent proves that it is not. But it is difficult to prove ergodicity analytically, and impossible to do so numerically.

In pace of calculations of ergodicity, we calculated the chaotic volume, ${ }^{9}$ i.e., the fraction of phase space for which the Lyapunov exponent is positive,

$\mu_{c}(E, \chi) \equiv \frac{\int d p_{1} d q_{1} d p_{2} d q_{2} \delta(E-H(\mathbf{q}, \mathbf{p}, \chi)) \Theta(\lambda(\mathbf{q}, \mathbf{p}))}{\int d p_{1} d q_{1} d p_{2} d q_{2} \delta(E-H(\mathbf{q}, \mathbf{p}, \chi))}$,

where

$$
\boldsymbol{\theta}(\boldsymbol{x}) \equiv \begin{cases}1, & x>0 \\ 0, & x \leq 0\end{cases}
$$

In practice the $\delta$ function in energy is replaced by a product of two step functions 


$$
\begin{aligned}
\delta(E-H(\mathbf{q}, \mathbf{p}, \chi)) \rightarrow & \Theta((E+\Delta E)-H(\mathbf{q}, \mathbf{p}, \chi)) \\
& \times \Theta(-(E-\Delta E)+H(\mathbf{q}, \mathbf{p}, \chi)) .
\end{aligned}
$$

We chose $\Delta E$ such that the energy range was divided into 25 energy bings, with a fivefold-finer binning around the energies -25.5 and 0 , i.e., at the onset of disappearance of chaos. The integral [Eq. (34)] was done by Monte Carlo methods. One hundred initial phase-space points were chosen at random in each energy range, the Lyapunov exponent was evaluated for each initial condition, and the exponent was judged to be positive or zero, thus yielding the fraction of points with positive exponents.

The heart of this integral is the calculation of the Lyapunov exponents. Here we used a Bulirsch-Stoer extrapolation method ${ }^{15}$ to integrate the equations of motion for $\mathbf{p}, q$ and the tangent vector $\eta$ [Eq. (31)]. If there is stretching in the phase space, $\boldsymbol{\eta}$ becomes quite large, so to avoid numerical overflows it was periodically rescaled to unity. Rescaling does not affect the time evolution of $\boldsymbol{\eta}$ since the equations of motion are linear. However, it does mean that we must keep track of the rescalings since the total growth or shrinkage of $\boldsymbol{\eta}$ is what we wish to calculate. ${ }^{21}$

The length of $\boldsymbol{\eta}$ was taken as the Euclidean norm - the choice of metric is irrelevant in the infinite-time limit. The appropriate time scale for each energy was defined as the average time between returns to a surface of section. The integration was then carried out for 100-250 units of time.

A difficulty arose in deciding which exponents are "zero" and which are positive. For this task the eye seemed better suited than the computer. The running Lyapunov exponent versus time was graphed on a log-log scale. The typical zero exponent decreases as $1 / t$, while a positive exponent remains constant. By looking at the graphs it was usually easy to separate out by eye the two different classes.

Figure 3 shows the final outcome of the calculation.

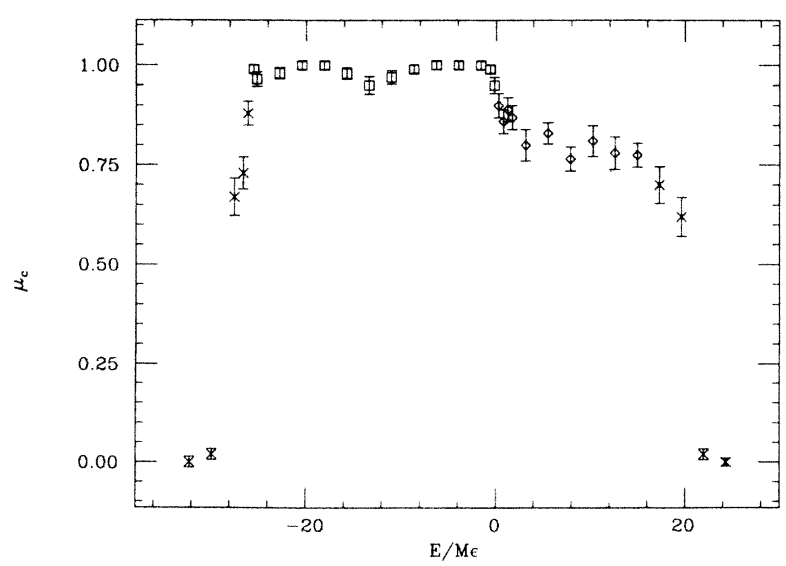

FIG. 3. Chaotic volume [Eq. (34)] for LMG model with $\chi=100$. The boxes indicate energies that were judged chaotic, the diamonds indicate quasichaotic energies, and the crosses show quasi-integrable energies.
There is a long energy range from -25.5 to -0.18 that is $95 \%$ chaotic or greater. These energies will be referred to as the chaotic energies, and the quantum states with eigenvalues in this range will be referred to as the chaotic states. The energies between -0.18 and 15 are $70-80 \%$ chaotic, and will be referred to as quasichaotic energies and the corresponding states are quasichaotic states. The remaining energies and the corresponding quantum states at both ends of the spectrum $(-33.34$ to -25.5 and 15 to 25.5) will be called quasi-integrable.

These three dynamics classes were chosen by considering both the classical dynamics and the need for good statistics. We would have preferred the classical dynamics to be the same (i.e., $\mu_{c}=$ const) within each class. Others achieved this goal by choosing systems that scale in energy ${ }^{8,9}$ (i.e., the dynamics are the same at all energies) but our model has no such scaling. In fact, we were fortunate to find a long range of energy for which the classical dynamics are chaotic. However, requiring that $\mu_{c}$ be nearly constant over the energy range of each class would have given too small an energy range, i.e., the number of eigenvalues in this range would not have been large enough to yield meaningful statistics. The final choice of dynamics classes was therefore a compromise between these two competing requirements.

The compromise was least for the chaotic levels: the change in $\mu_{c}$ is smallest in that energy range. Since the classical dynamics are not stationary for the quasichaotic and quasi-integrable levels, we can expect only qualitative, not quantitative, agreement with the predictions for quantum systems whose classical analog has intermediate dynamics. ${ }^{9,11}$

There is some suggestion that the system at the chaotic energies may be ergodic: the Lyapunov exponents for all the Monte Carlo points look as though they are converging to the same value (Fig. 4), and the points on the sur-

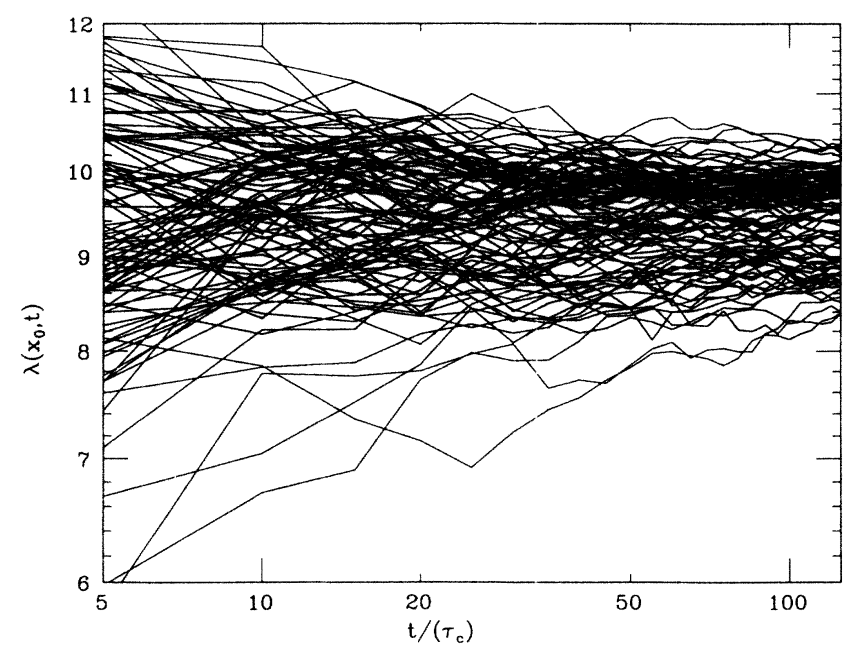

FIG. 4. Lyapunov exponent [Eq. (33)] at finite time for $\chi=100,-21.6 \leq E \leq-19.3$ and 100 initial conditions. It appears as though $\lambda\left(\mathbf{x}_{0}, t\right)$ for all $\mathbf{x}_{0}$ may be converging to the same value; this result suggests ergodicity. The time constant $\tau_{c}$ is the average time between intersections of a trajectory with a fixed plane in phase space. 
face of section for one trajectory evenly cover the available phase space (Fig. 2). These are necessary but not sufficient to prove ergodicity.

\section{DENSITY OF STATES}

Random matrix theory focuses on statistics of spectral fluctuations; these can be defined only relative to some smooth behavior. In this section we discuss the separation between the average and the fluctuation properties of the spectrum.

A spectrum is defined by the staircase function $N(E)$ that gives the number of eigenvalues with energy less than $E$. We can write this as the sum of two parts,

$$
N(E)=N_{\mathrm{av}}(E)+N_{\text {fluct }}(E) .
$$

The separation between the smoothed average behavior and the fluctuations from that behavior is somewhat arbitrary, but should be done in an unbiased manner.

The smooth behavior is nonuniversal, ${ }^{3,4}$ and therefore cannot be modeled by RMT. It must be removed before comparisons between spectra can be made. This is done by "unfolding" the spectrum via the mapping

$$
x_{i} \equiv N_{\mathrm{av}}\left(E_{i}\right) \text {. }
$$

We calculate $N_{\mathrm{av}}(E)$ for the LMG model from $\rho_{\mathrm{av}}(E)$ (the average density of states) which is in turn obtained from the Weyl formula,

$$
\begin{aligned}
\rho_{\mathrm{av}}(E) & \equiv \rho_{\text {semiclassical }}(E, \chi) \\
& \propto \int d^{N} q d^{N} p \delta(E-H(\mathrm{q}, \mathrm{p}, \chi)),
\end{aligned}
$$

which says that the number of states is proportional to the classical phase-space volume.

Because we know the eigenvalues for $\chi=0$, we can calculate the density of states $\rho(E, \chi=0)$ in analytic form. In this limit the eigenvectors in the symmetric representation are denoted by the number of particles in each orbital. The energy of the lowest state is -1 , obtained uniquely when all particles are in the lower orbital; the highest energy is 1 , and is also nondegenerate. The populations for states with energy $n / M$ can take the values $\left(\frac{1}{2}(M-m-n), m, \frac{1}{2}(M+n-m)\right)$ with $m=M-|n|$, $M-|n|-2, \ldots, 1$ or 0 . This shows that $\rho$ varies linearly with $|n|$, and in the large- $M$ limit we thus obtain for the normalized density [ $\left.\int \rho(E) d E=1\right]$,

$$
\rho(E, 0)= \begin{cases}1-E, & E>0 \\ 1+E, & E<0 .\end{cases}
$$

The symmetry about zero reflects a symmetry in the Hamiltonian: the energy is antisymmetric under interchange of populations in orbitals 0 and 2 . The interaction destroys this symmetry because that term is symmetric under interchange of the population of any two orbitals.

To calculate the level density when $\chi$ is not zero, we performed a Monte Carlo integration of Eq. (39), where the $\delta$ function was again replaced by a product of two step functions [see Eq. (36)] with $\Delta E$ chosen to give 200 bins. Because the energy is invariant under reversal of the sign of any two canonical variables, we needed only to choose points from two of the 16 phase-space sectors. The result for $\chi=100$ is shown in Fig. 5. For this value of $\chi$ the symmetry about $E=0$ has disappeared as expected.

We obtained an alternative $\rho_{\text {av }}$ by histogramming the 7380 eigenvalues for $M=120$ using 50 bins (Fig. 5) and then smoothing with cubic splines. This second method allowed a check of the dependence of spectral fluctuations on the unfolding. The two different density of states are denoted by $\rho_{\mathrm{av}}^{\text {class }}$ and $\rho_{\mathrm{av}}^{\text {quant }}$.

\section{SPECTRAL FLUCTUATIONS}

We examined the LMG spectral averages of five fluctuation measures and compared them to the ensemble averages of the GOE and Poisson ensemble. These measures are calculable with a relatively small number of levels and the results are quite different for the Poisson and GOE ensemble averages. The calculations shown were done for $M=120$ and $\chi=100$. The number of levels in each signature and classical dynamics class are given in Table II. The quasi-integrable levels at low energies (i.e., $E \approx-33$ ) were not used [except for $P(s)$ ] since in all classes this interval contains only 50 levels - too few to give meaningful statistics.

The first statistics is the nearest-neighbor spacing distribution, the probability that two neighboring eigenvalues are a distance $s$ apart. The Poisson ensemble average is $P(s)=e^{-s, 3,24}$ which gives a large probability for the occurrence of near degeneracies, while the GOE average is closely approximated by the Wigner distribution

$$
P(s)=(\pi / 2) s \exp \left[-(\pi / 4) s^{2}\right]
$$

(Refs. 3 and 25), which gives level repulsion.

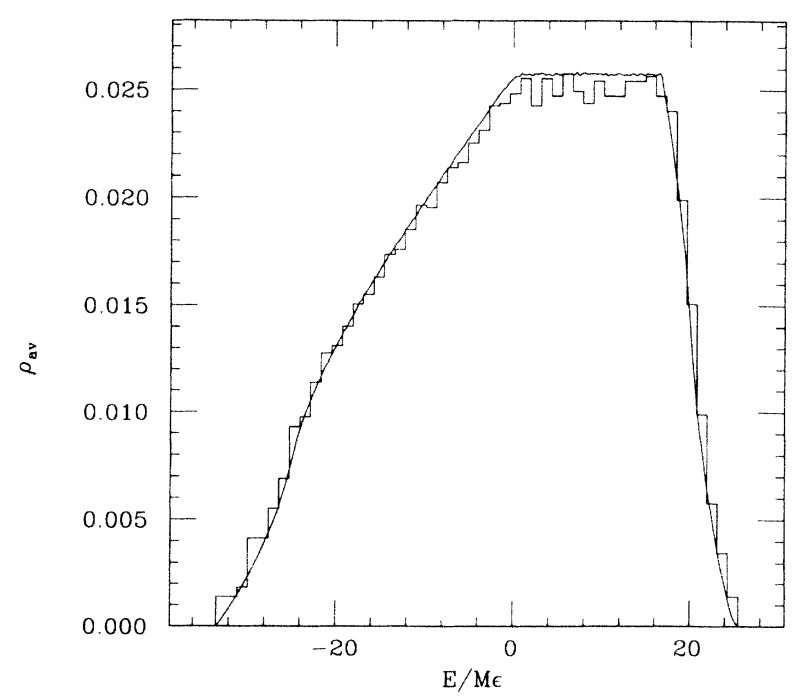

FIG. 5. Density of states from classical and quantum calculations, normalized so that the total number of states is 1 . The histogram shows the number of eigenstates in each energy interval for $M=120$; the total number of states is 7380 . The smooth curve is the semiclassical result obtained from evaluating Eq. (39). 
TABLE II. Number of quantum states for $M=120$ and $\chi=100$ in each dynamics and signature class.

\begin{tabular}{lllll}
\hline \hline & eee & eoo & oeo & ooe \\
\hline Chaotic & 835 & 807 & 806 & 805 \\
Quasichaotic & 718 & 696 & 697 & 696 \\
Quasi-integrable & 283 & 273 & 273 & 275 \\
\hline
\end{tabular}

In terms of the unfolded spectrum, the spacings were calculated as follows:

$$
s_{i} \equiv x_{i+1}-x_{i},
$$

and then histogrammed to give $P(s)$. The qualitative behavior of the results is the same, whether we use $\rho_{\mathrm{av}}^{\text {class }}$ or $\rho_{\mathrm{av}}^{\text {quant }}$ in the unfolding procedure. We checked the stationarity of $P(s)$ by dividing the chaotic levels into three subintervals, each with about 280 levels. The statistics for each subinterval were in agreement with the Wigner distribution. The results were also independent of the signature class.

The final results were obtained by combining $P(s)$ for all four signature classes after the spacings were calculated in each class separately (Fig. 6). The chaotic levels were in good agreement with the GOE average, while the

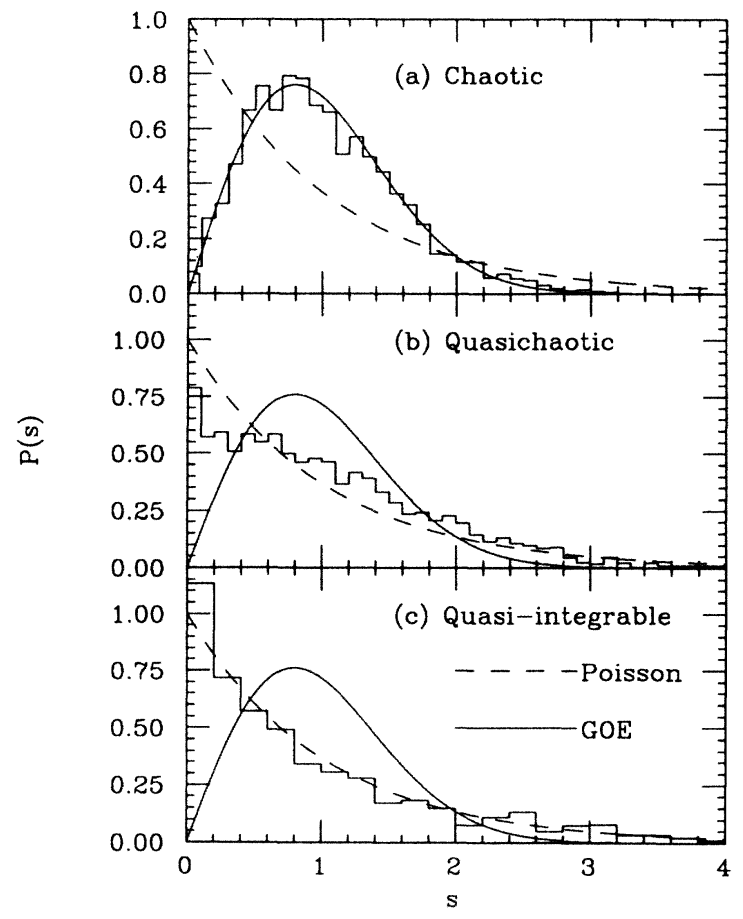

FIG. 6. Histograms of the nearest-neighbor spacing $s$ [Eq. (41)], where $s_{i}$ 's were calculated for each signature class separately and then combined. Panel (a) shows the 3249 spacings from the chaotic levels, panel (b), the 2803 spacings from quasichaotic levels, and panel (c), the 1100 spacings from quasiintegrable levels. As hypothesized, the chaotic levels agree with GOE, the quasi-integrable levels agree with Poisson, and the quasichaotic levels lie in between. quasi-integrable levels fit the Poisson distribution, and the quasichaotic levels fell in between. This progression is in qualitative agreement with Seligman et al. ${ }^{9}$ and Berry and Robnik. ${ }^{11}$ Specifically, we see that for the quasichaotic levels, $P(s)$ shows large numbers of near degeneracies.

The $\Delta_{3}$ statistic measures spectral rigidity. This is defined by $y^{3,4}$

$\Delta_{3}(\alpha, L) \equiv \min _{A, B} \frac{1}{L} \int_{\alpha}^{\alpha+L}[N(x)-(A x+B)]^{2} d x$,

where $N(x)$ is defined above Eq. (37). A small value of $\Delta_{3}(L)$ indicates that the spectrum is stiff: given one eigenvalue one can predict the location of distant eigenvalue with some accuracy. A large value of $\Delta_{3}(L)$ means that such a prediction cannot be made with confidence. A GOE spectrum is much stiffer than a Poisson spectrum: $\bar{\Delta}_{3}(L) \approx \pi^{-2} \ln L-0.0007$ ( $\left.L \gtrsim 15\right)$ for GOE and $L / 15$ for Poisson (the bar indicates the ensemble average). ${ }^{3}$

To calculate $\Delta_{3}$ for the LMG spectra, we used the numerically efficient formula ${ }^{3}$

$$
\begin{aligned}
\Delta_{3}(\alpha, L)= & \frac{n^{2}}{16}-\frac{1}{L^{2}}\left[\sum_{i=1}^{n} \widetilde{x}_{i}\right]^{2}+\frac{3 n}{2 L^{2}}\left[\sum_{i=1}^{n} \widetilde{x}_{i}^{2}\right] \\
& -\frac{3}{L^{4}}\left[\sum_{i=1}^{n} \tilde{x}_{i}^{2}\right)^{2}+\frac{1}{L}\left[\sum_{i=1}^{n}(n-2 i+1) \widetilde{x}_{i}\right),
\end{aligned}
$$

where $\tilde{x} \equiv x-(\alpha+L / 2)$ are the shifted, unfolded eigenvalues and $n$ is the number of levels in the interval $[\alpha, \alpha+L]$. For comparison with ensemble averages, we calculated the spectral average

$$
\left\langle\Delta_{3}(L)\right\rangle \equiv \frac{1}{N_{\alpha}} \sum_{\alpha} \Delta_{3}(\alpha, L) .
$$

where the $\alpha$ 's were chosen such that successive intervals overlap by $L / 2$ and $N_{\alpha}$ counts the number of intervals. This choice was made to balance the desire for good statistics and the need to avoid correlations.

The last three statistics that we consider are cumulants of the number statistic $n(\alpha, L)$ which gives the number of levels in the energy interval $[\alpha, \alpha+L]$. Because the spectra are unfolded, $\langle n(L)\rangle=L$ for all spectra. However, the cumulants of the number statistic will in general be different for different spectra. These spectral cumulants, denoted by the $s$ subscript, are defined as follows:

$$
\begin{aligned}
& \Sigma_{s}^{2}(L) \equiv\left\langle[n(\alpha, L)-\langle n(L)\rangle]^{2}\right\rangle, \\
& \gamma_{1, s}(L) \equiv \frac{\left\langle[n(\alpha, L)-\langle n(L)\rangle]^{3}\right\rangle}{\Sigma_{s}^{3}(L)}, \\
& \gamma_{2, s}(L) \equiv \frac{\left\langle[n(\alpha, L)-\langle n(L)\rangle]^{4}\right\rangle}{\Sigma_{s}^{4}(L)}-3,
\end{aligned}
$$

known as the variance, skewness, and excess (or kurtosis), respectively. The appearance of powers of the variance in the denominator of $\gamma_{1}$ and $\gamma_{2}$ gives these third and fourth cumulants in terms of a natural scale. Note that 
both $\gamma_{1}$ and $\gamma_{2}$ are zero for a Gaussian distribution.

For comparison, we need to know the GOE and Poisson ensemble averages, which are listed in Table III. ${ }^{3}$ While the Poisson results can be expressed analytically, not all the GOE results are known in closed form. The GOE number variance (with the ensemble averaged cumulants denoted by the $e$ subscripts) is given exactly by ${ }^{6}$

$$
\begin{aligned}
\Sigma_{e}^{2}(L)=\frac{2}{\pi}[ & \ln (2 \pi L)+\gamma+1+\frac{1}{2}[\operatorname{Si}(\pi L)]^{2} \\
& -\frac{1}{2} \pi \operatorname{Si}(\pi L)-\cos (2 \pi L)-\operatorname{Ci}(2 \pi L) \\
& \left.+\pi^{2} L\left[1-\frac{2}{\pi} \operatorname{Si}(2 \pi L)\right]\right],
\end{aligned}
$$

where $\mathrm{Ci}$ and $\mathrm{Si}$ are the sine and cosine integrals and $\gamma$ is Euler's constant. The other statistics can be expressed in terms of the variance and the ensemble-averaged spacing distributions $E_{e}(k, l){ }^{3}$

$$
\begin{aligned}
& \gamma_{1, e}(L)=\sum_{k=0}^{\infty} \frac{(k-L)^{3} E_{e}(k, L)}{\Sigma_{e}^{3}(L)}, \\
& \gamma_{2, e}(L)=\sum_{k=0}^{\infty} \frac{(k-L)^{4} E_{e}(k, L)-3 \Sigma_{e}^{2}(L)}{\Sigma_{e}^{4}(L)}, \\
& \bar{\Delta}_{3}(L)=\frac{2}{L^{4}} \int_{0}^{L}\left(L^{3}-2 L^{2} x+x^{3}\right) \Sigma_{e}^{2}(x) d x .
\end{aligned}
$$

The values of $E_{e}(k, L)$, the probability that an interval of length $L$ contains exactly $k$ levels, are tabulated in Ref. 26. The sum over $k$ was actually taken only over seven values at most, since $E_{e}(k, L)$ is strongly peaked at $k=L$.

There are errors in the spectral averages due to the finite-sample size. We can determine the error either from the sample, or from the ensemble distribution itself. For $\Delta_{3}$ we determined the error from the sample

$$
\operatorname{var}\left[\Delta_{3}(L)\right] \equiv\left\langle\left[\Delta_{3}(\alpha, L)-\left\langle\Delta_{3}(L)\right\rangle\right]^{2}\right\rangle .
$$

This variance is shown in the graphs of the results.

For the $r$ th moment of the number statistic, the variance is given by

$$
\operatorname{var}\left(m_{r}\right) \approx \frac{1}{N_{\alpha}}\left(m_{2 r}-m_{r}^{2}\right),
$$

and depends on higher moments. For our small sample sizes, the variances of the moments are not well determined. We therefore turned to ensemble averages for estimates of errors due to finite-sample sizes. We quote the results from Bohigas et al. ${ }^{27}$ They used Monte Carlo techniques to construct many members of the GOE, and then performed ensemble averaging. Their results for a

TABLE III. GOE and Poissons ensemble averages for cumulants of the number statistic (Ref. 3 ). There are no analytic expressions for the skewness and excess of the GOE.

\begin{tabular}{lcccc}
\hline \hline & $\Sigma_{e}^{2}(L)$ & $\gamma_{1, e}(L)$ & $\gamma_{2, e}(L)$ \\
\hline GOE & $\left(2 \pi^{2}\right) \ln L+0.44$ & $(L \gtrsim 1)$ & & \\
Poisson & $L$ & & $1 / \sqrt{L}$ & $1 / L$ \\
\hline \hline
\end{tabular}

sample size of 1762 are given in Table IV. Since our sample size is smaller, these give a lower bound on the errors. The variance vanishes at least as quickly as $1 / p$, where $p$ is the sample size. ${ }^{28}$ Therefore, we can adjust these error estimates by multiplying by $\sqrt{1762 / p}$. This factor ranges from 2.5 for $p=280$ to 1.45 for $p=835$.

Now we turn to our results. Again we checked that they are independent of the unfolding procedure. Using either $\rho_{\mathrm{av}}^{\text {quant }}$ or $\rho_{\mathrm{av}}^{\text {class }}$ gave the same $\Delta_{3}$ results, but $\Sigma_{s}^{2}$, $\gamma_{1, s}$, and $\gamma_{2, s}$ disagreed for $L \gtrsim 2.5$. This is a manifestation of the errors due to the small-sample size; the fluctuations were of the size predicted by GOE (Table IV). We also checked stationarity, again by dividing the chaotic levels into three intervals. We did see some dependence of $\Delta_{3}$, but this was to be expected. We know that the classical dynamics for over the chaotic energies are not completely independent of energy: $\mu_{c}$ varies between 0.95 and 1.00. We found that the most chaotic levels $\left(\mu_{c}=1.0\right)$ gave the stiffest spectrum, while the least chaotic $\left(\mu_{c} \approx 0.97\right)$ gave the softest. The higher statistics also showed stationary behavior for $L \leqslant 1.5$. However, again we saw the finite-sample effects in the scatter at higher $L$, and since the sample is smaller the variations were visibly greater.

Looking at the oeo class, we examined the behavior for chaotic, quasichaotic, and quasi-integrable levels (Fig. 7). Here, as in the subsequent figures, the GOE averages are shown by the solid curves and Poisson predictions are given by the dashed curves. We see the predicted results: good agreement with GOE for the chaotic levels, a less stiff spectrum for the quasichaotic levels, and an even less stiff spectrum for the quasi-integrable levels. For the $\gamma_{1, s}$ and $\gamma_{2, s}$ statistics of the quasi-integrable levels there is significant systematic deviation from both GOE and Poisson results.

However, we found completely unexpected results when we checked the dependence on signature class (Fig. 8). For $M=120$, eee was significantly softer than ooe and eoo which were in turn softer than oeo. When we looked at an odd- $M$ matrix, the pattern was repeated, but with all even and odd labels exchanged (i.e., eoe was stiffest, ooo least stiff). In the following discussion of this phenomenon we keep to $M$ even, but the conclusions also apply to the $M$-odd case. We also examine only the chaotic levels, since this gives the largest number of levels and the classical dynamics is nearly stationary.

The explanation became clear when we examined the $\chi=\infty$ limit. In this case the three single-particle orbitals are degenerate in energy, and $H$ is invariant under interchange of the orbital labels [Eqs. (6) and (7)]. Let $P_{i j}$ be

TABLE IV. GOE averages for variances of moments of the number statistic for a sample including 1762 levels, obtained from Ref. 27.

\begin{tabular}{cccc}
\hline \hline$L$ & $\operatorname{var}\left[\Sigma^{2}(L)\right]$ & $\operatorname{var}\left[\gamma_{1}(L)\right]$ & $\operatorname{var}\left[\gamma_{2}(L)\right]$ \\
\hline 0.25 & 0.001 & $\approx 0.02$ & 0.08 \\
$\approx 1$ & 0.009 & $\approx 0.02$ & 0.05 \\
5 & 0.03 & 0.05 & 0.08 \\
\hline \hline
\end{tabular}


the operator that permutes orbitals $i$ and $j$. Then $H P_{i j}=P_{i j} H$. In the classical system, this discrete symmetry means that $H$ is unchanged if $p_{1} \leftrightarrow p_{2}$ and $q_{1} \leftrightarrow q_{2}$. A discrete symmetry does not induce the integrability associated with a continuous symmetry, so the system can still be chaotic. However, in the quantum system, a discrete symmetry implies that $H$ can be written in block-diagonal form. If we look at the spectrum of the entire matrix, we should expect to see Poisson statistics ${ }^{3,4}$ regardless of the classical dynamics. However, if we look at the spectra of each of the block-diagonal matrices separately, we should see GOE results if the system is classically chaotic, or Poisson if it is classically integrable.

For the $\chi=100$ case, we are quite close to the $\chi=\infty$ limit. Therefore we have a "partially conserved" discrete symmetry. This makes no difference to the classical dynamics, but in the quantum system $H$ can be written in nearly block-diagonal form, "nearly" meaning that the average matrix element connecting these blocks is relatively small.

To see if this partially conserved discrete symmetry
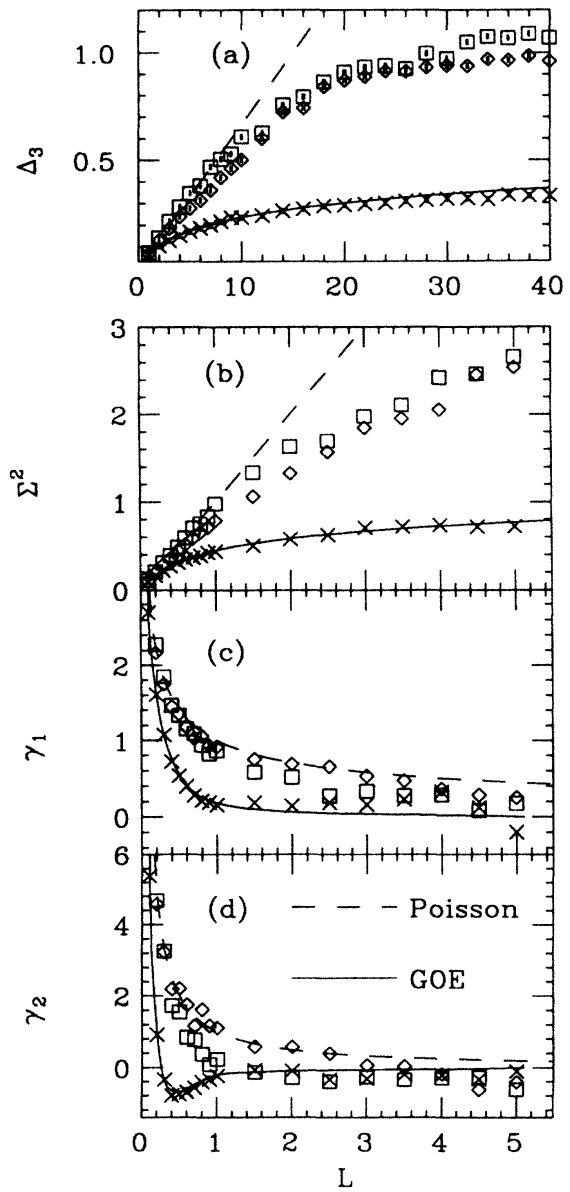

FIG. 7. Spectral statistics for the oeo signature class and the three different dynamics classes. The statistics of the 835 chaotic states (indicated by crosses) are in agreement with GOE, while those of the 718 quasichaotic (indicated by diamonds) and of the 283 quasi-integrable (indicated by squares) states are approaching Poisson. was the cause of dependence on signature, we calculated the eigenvalues for $\chi=\infty$. (We implement this limit in practice by setting $\varepsilon=0$ and $\chi=1$.) There are only two signature classes (and therefore only two different spectra) in this limit since the oeo, ooe, and eoo matrices are identical. The eigenvalues were calculated twice, once for the entire matrix, and once for the block-diagonal matrices separately.

To bring $H$ into block-diagonal form, we needed new basis states. This is easiest for the eoo signature class. Because $s_{0}$ is even while $s_{1}$ and $s_{2}$ are odd, we need only construct states that have a definite parity under interchange of labels 1 and 2. Using the states defined in Eq. (4), and showing the population of the ground state explicitly, we write the new basis,

$$
\begin{aligned}
& \Psi_{\text {sym }} \equiv \frac{1}{\sqrt{2}}(|a, b, c\rangle+|a, c, b\rangle), \\
& \Psi_{\text {asym }} \equiv \frac{1}{\sqrt{2}}(|a, b, c\rangle-|a, c, b\rangle) .
\end{aligned}
$$

Because $H$ does not couple these two bases, $H_{\text {eoo }}$ decom-
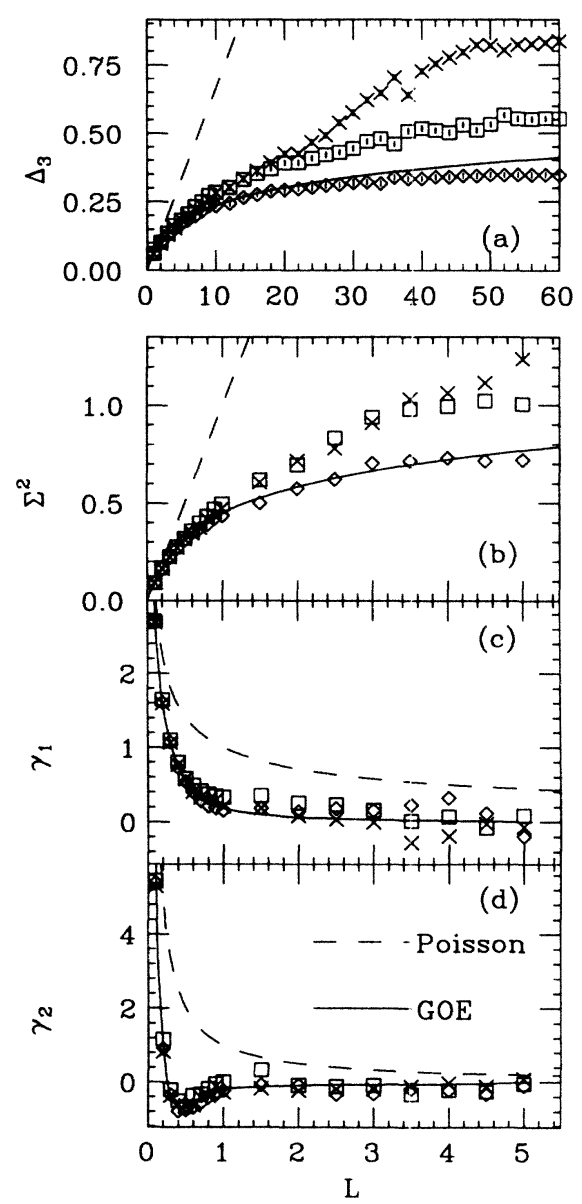

FIG. 8. Spectral statistics for the chaotic dynamics class for three different signature classes. The oeo statistics are denoted by diamonds, the eee class denoted by crosses, and the eoo class denoted by squares. The ooe class is omitted for clarity, since its spectral statistics are quite close to those of the eoo class. 
poses into two blocks. We diagonalized $H$ in each basis separately.

The eee class is more complicated since the populations of all the orbitals can be interchanged. The basis states are

$$
\begin{aligned}
& \Psi_{\mathrm{sym}} \equiv \frac{1}{\sqrt{6}}(|a, b, c\rangle+|b, a, c\rangle+|b, c, a\rangle \\
& +|c, b, a\rangle+|c, a, b\rangle+|a, c, b\rangle), \\
& \Psi_{\text {asym }} \equiv \frac{1}{\sqrt{6}}(|a, b, c\rangle-|b, a, c\rangle+|b, c, a\rangle \\
& -|c, b, a\rangle+|c, a, b\rangle-|a, c, b\rangle), \\
& \Psi_{P_{01}, \mathrm{sym}}^{(1)} \equiv \frac{1}{\sqrt{12}}(2|a, b, c\rangle+2|b, a, c\rangle-|b, c, a\rangle \\
& -|c, b, a\rangle-|c, a, b\rangle-|a, c, b\rangle), \\
& \Psi_{P_{01}, \text { sym }}^{(2)} \equiv \frac{1}{\sqrt{4}}(|b, c, a\rangle+|c, b, a\rangle-|c, a, b\rangle-|a, c, b\rangle), \\
& \Psi_{P_{01}, \text { asym }}^{(1)} \equiv \frac{1}{\sqrt{12}}(2|a, b, c\rangle-2|b, a, c\rangle-|b, c, a\rangle \\
& +|c, b, a\rangle-|c, a, b\rangle+|a, c, b\rangle), \\
& \Psi_{P_{01}, \text { asym }}^{(2)} \equiv \frac{1}{\sqrt{4}}(|b, c, a\rangle-|c, b, a\rangle-|c, a, b\rangle \\
& +|a, c, b\rangle) \text {. }
\end{aligned}
$$

The first two states are totally symmetric or antisymmetric under interchange of any two orbitals. The states $\Psi_{P_{01} \text {,sym }}$ are symmetric only under interchange of orbitals 0 and 1 , while $\Psi_{P_{01} \text {, asym }}$ are antisymmetric under the same operation; all four $P_{01}$ states have mixed parity under any of the other permutation operations. In this basis $H$ couples only $P_{01}$ symmetric states together and the $P_{01}$ antisymmetric states together; therefore, the eee matrix is made up of four blocks.

We also needed to calculate $\rho_{\mathrm{av}}^{\text {class }}$ and $\mu_{c}(\chi=\infty)$ in order to check the correlations between classical dynamics and spectral statistics. The results, except for energy scale, are very similar to the $\chi=100$ values. The classically chaotic energies lie in the interval $[-0.242$, $0.0159]$.

Figure 9 shows $\Delta_{3}$ and $\Sigma_{s}^{2}$ for $\chi=\infty$ and the eoo signature class. The statistics of the entire eoo or eee class are much closer to Poisson than GOE. However, the spectra of the individual symmetry classes separately are in good agreement with GOE. The results are independent of permutation symmetry class and signature class.

For the $\chi=100$ case, we can understand qualitatively the relative stiffness of the spectra, as indicated by the $\Delta_{3}$ results. The eoo and ooe spectra are nearly the same, and stiffer than the eee class. If we treat the energy separation of the three single-particle orbitals as a perturbation, then the matrix element mixing symmetric and antisym- metric states is proportional to $\varepsilon$ for eoo and ooe and proportional to $2 \varepsilon$ for oeo. Therefore in the ooe and eoo classes the symmetry is "less broken" than in the oeo case. The eee class is the softest of all; we may attribute this to the difference between having two or four blocks for $\chi=\infty$. The eee class has four, so the matrix has relatively more zeros, and therefore less coupling, to begin with. When the perturbation is added, although the size of the mixing is the same as for the other classes, its effect is diluted.

The classical Hamiltonian does not reflect the signature of the quantum states, and hence cannot account for the signature dependence. We could write [Eqs. (2) and (9)]

$$
\begin{aligned}
\Psi_{x e e} & \equiv\left(e^{z_{1} G_{10}}+e^{-z_{1} G_{10}}\right)\left(e^{z_{2} G_{20}}+e^{-z_{2} G_{20}}\right)|0\rangle \\
& =\left|z_{1}, z_{2}\right\rangle+\left|-z_{1}, z_{2}\right\rangle+\left|z_{1},-z_{2}\right\rangle+\left|-z_{1},-z_{2}\right\rangle,
\end{aligned}
$$

where the subscript xee indicates that this state has an even number of particles in orbitals 1 and 2, while the sig-
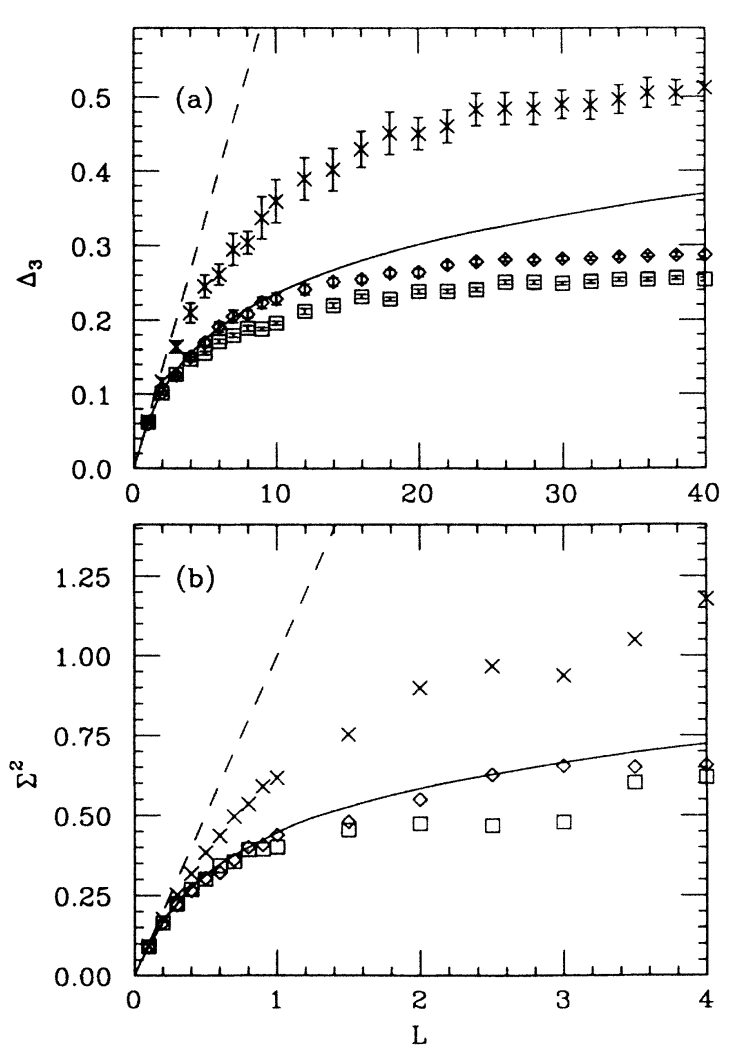

FIG. 9. $\Delta_{3}$ and $\Sigma^{2}$ statistics for $\chi=1$ and $\varepsilon=0$, the eoo signature class, and the chaotic dynamics class. The crosses indicate the results for the entire spectrum, the diamonds indicate those for the symmetric spectrum [Eq. (50)] only, and the squares indicate those for the antisymmetric spectrum only. The statistics are in better agreement with GOE averages when the symmetries are respected. 
nature of the ground orbital is unknown until $M$ is specified. We could also write

$$
\begin{aligned}
\Psi_{x 00} & \equiv\left(e^{z_{1} G_{10}}-e^{-z_{1} G_{10}}\right)\left(e^{z_{2} G_{20}}-e^{-z_{2} G_{20}}\right)|0\rangle \\
& =\left|z_{1}, z_{2}\right\rangle-\left|-z_{1}, z_{2}\right\rangle-\left|z_{1},-z_{2}\right\rangle+\left|-z_{1},-z_{2}\right\rangle,
\end{aligned}
$$

with similar expressions for $\Psi_{x e o}$ and $\Psi_{x o e}$. In the $M \rightarrow \infty$ limit, the expectation value of $H$ between coherent states of different $z$ vanishes. Hence the cross terms in expectation values evaluated with the states (52) or (53) disappear, and the classical limit is independent of the signature class. This is as we would expect; in the $M \rightarrow \infty$ limit, evenness and oddness are of no consequence.

Finally, we examined the saturation of the $\Delta_{3}$ statistic (i.e., the flattening out at a finite $L$, see Fig. 10) which can be explained by semiclassical arguments, but not by RMT. The explanation was given by Berry ${ }^{12}$ and is based on the work of Gutzwiller ${ }^{29}$ and Balian and Bloch. ${ }^{30}$ This approach expresses the fluctuations of the spectrum in terms of the actions for classical periodic trajectories. Berry writes the density of states

$$
\rho(E)=\rho_{\mathrm{av}}(E)+\rho_{\text {fluct }}(E),
$$

where

$$
\rho_{\text {fluct }}(E) \equiv \sum_{j} A_{j}(E) \exp \left[i S_{j}(E) / \hbar\right]
$$

Here $j$ labels all periodic trajectories, $S_{j}$ is the action along trajectory $j$, and $A_{j}$ is an amplitude associated with this trajectory. From this expression for $\rho$, Berry derives an expression for $\Delta_{3}(L)$. The essential point here is that periodic orbits with periods $T_{j}<\hbar \rho_{\mathrm{av}} / L$ do not contribute to $\Delta_{3}(L)$ because the action of these orbits does not vary much over $L$. Let $T_{\min }$ be the period of the shortest

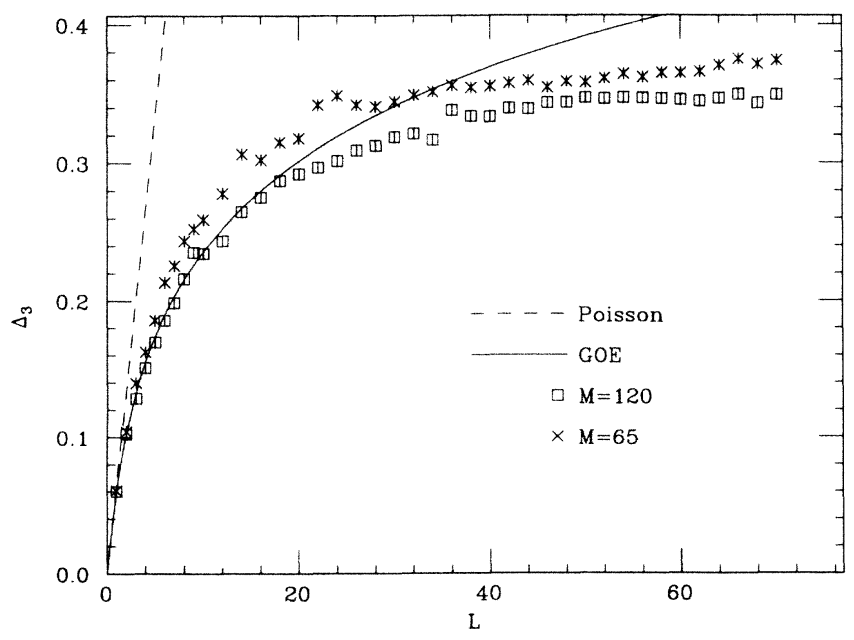

FIG. 10. Saturation of the $\Delta_{3}(L)$ statistic occurs at $L \approx 20$ for $M=65$, and at $L \approx 40$ for $M=120$. This scaling of $L$ with $M$ agrees with the prediction of Berry (Ref. 12) [Eq. (58)]. orbit. If

$$
L \geq L_{\max } \equiv \frac{\hbar \rho_{\mathrm{av}}}{T_{\min }},
$$

then all periodic orbits contribute to $\Delta_{3}(L)$ and, as a result, $\Delta_{3}(L)$ saturates at $L_{\max }$.

To compare with Berry's prediction, we must relate $\hbar$ to $M$. By the Weyl rule, the number of states $\mathcal{N}$ is

$$
\mathcal{N} \equiv \frac{V_{\text {phase space }}}{4 \pi^{2} \hbar^{2}}
$$

For the LMG model the total number of symmetric states is $\approx M^{2} / 2$. The phase-space volume $V_{\text {phase space }}$ is that of a four-ball of radius $\sqrt{2}$. Combining these last three expressions gives $\hbar=\sqrt{2} / M$, which is consistent with the discussion following Eq. (19). The density of states is the product of the normalized density of states, which is about 0.02 for the chaotic energies (see Fig. 5), and the size of the basis (approximately $M^{2} / 8$ ). Inserting these values into the definition of $L_{\max }$, we have

$$
L_{\max }=\frac{0.0035 M}{T_{\min }} \text {. }
$$

Note that $L_{\max }$ scales linearly with $M$. In Fig. 10 we show $\Delta_{3}$ for $M=65$ and $M=120$. For $M=120$ the statistic saturates at $L \approx 40$. For $M=65$, it does not appear as though saturation even occurs; however, the rate of increase becomes a relatively small constant at $L \approx 20$. If we take this as the saturation point, the $M$ scaling holds very well.

Using $L_{\max }=40$ and $M=120$, we obtain $T_{\min }=0.01$. This is about $\frac{1}{7}$ the characteristic time, i.e., the time between crossings of a fixed plane in phase space. This is smaller than might be expected, but not totally unreasonable.

This rough agreement is all we can hope for, both because we are far from the semiclassical limit where the analytical results apply [specifically, $\rho(E)$ is not constant over the chaotic energies] and because we know nothing about the periodic orbits of the classical system.

\section{OVERLAP DISTRIBUTIONS}

RMT describes eigenvector statistics as well as eigenvalue statistics. In this section we compare the eigenvector statistics of the LMG model with those of the GOE.

For typical members of the GOE, the overlap

$$
x_{i \lambda} \equiv\langle i \mid \lambda\rangle
$$

is a Gaussian random variable, where $i$ is an eigenvector and $\lambda$ is an arbitrary basis state. ${ }^{4}$ For ensembles with finite $\mathcal{N}$, the width of the Gaussian distribution is $1 / \sqrt{\mathcal{N}}$, where $\mathcal{N}$ is the size of the basis; this is simply the rootmean-square overlap for normalized states.

There is no quantitative prediction for the overlaps of an integrable system. However, we expect that an eigenstate of an integrable system is expressed in terms of only a few states of some fixed basis. Therefore, the distribution should have a few very large overlaps (with the constraint that $\left|x_{i \lambda}\right| \leq 1$ ) and many very small overlaps (in 
keeping with the constraint $\sum_{i} x_{i \lambda}^{2}=1$ ).

Calculation of the LMG eigenvectors is relatively easy: the Hilbert space is compact so we can calculate all the eigenvectors without ad hoc truncation of the basis. This is a considerable advantage of our model; most systems that have been studied by others do not yield many reliable eigenstates. All of the results in this section are for $M=85$, for which $\mathcal{N}_{\text {ooo }}=903$ and $\mathcal{N}_{\text {eeo }}=\mathcal{N}_{\text {oee }}=\mathcal{N}_{\text {eoe }}$ $=946$.

Our choice of basis states was the original basis in which the Hamiltonian is written, i.e., the eigenstates of the number operators. To compare with the GOE average, we histogrammed $x_{i \lambda}$ to give the probability distribution $P\left(x_{i \lambda}\right)$, allowing $\lambda$ to take on all possible values and letting $i$ range over all values in a particular dynamics class. The results were independent of signature class. All dynamics classes showed the same qualitative behavior (Fig. 11): an excess of very small overlaps and of very

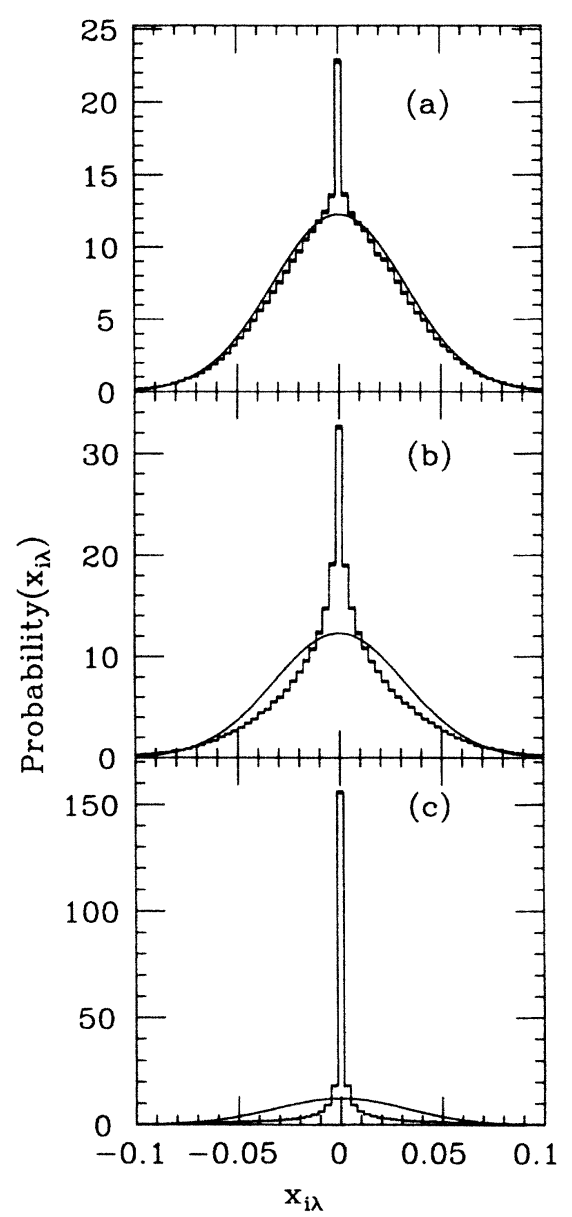

FIG. 11. Histograms of the overlap [Eq. (59)] of eigenvectors of the LMG model ( $\chi=100$ and eoe signature class) with a set of basis vectors [Eq. (4)]. Panel (a) shows the distribution when the overlap is taken with the chaotic levels, panel (b) shows the same results for the quasichaotic levels, and panel (c), the results for the quasi-integrable levels at the high end of the spectrum. The histograms include 393480,337 546, and 137370 counts, respectively. The solid line is the GOE prediction: a Gaussian of width $1 / \sqrt{\mathcal{N}}$. large overlaps relative to a Gaussian distribution. However, the degree of the excess varies significantly: it is largest for the quasi-integrable levels and smallest for the chaotic levels.

We then broke the chaotic levels into six nearly equal groups (Fig. 12). Although the chaotic volume is nearly constant over this energy interval (Fig. 3), the overlap probability distribution varies significantly. The best agreement is found for $E=[-6.6,-3.3]$ but even here it is clear that the excess of small overlaps is real, and not due to statistical fluctuations. Deviations from GOE have also been seen by Heller. ${ }^{31}$

To first order, we see good agreement with the GOE averages for the chaotic overlaps, yet this is clearly not the whole story. We must answer why the results are not correlated with the chaotic volume, and why the eigenvector overlaps are only in fair agreement with GOE averages, while the eigenvalue statistics are in good agreement. At the present time we only have speculation to offer.

In answer to the first question, we acknowledge that the chaotic volume may not be the best indicator of the character of the classical dynamics. As an alternate chaotic parameter, we calculated the average rate of exponential separation, which is given by the Lyapunov exponent averaged over phase space. This parameter also failed to correlate with $P\left(x_{i \lambda}\right)$. It may be the case that the eigenvectors are sensitive to the details of phase space (e.g., whether the KAM tori exist only in one small region or are sprinkled throughout phase space) or some other feature which we have not considered.

To answer the second question, we suggest that eigenvectors should be much more sensitive indicators of chaos than are the eigenvalues. This is because, to obtain a Gaussian distribution, there must be no constraints that prevent any eigenvector from overlapping with any basis vector. This requires a degree of mixing in Hilbert space that is not obviously necessary to obtain GOE spectral fluctuations.

Finally, we should also consider the possibility that the partially conserved discrete symmetry discussed in Sec. V may have an effect on the overlaps as well as the spectral statistics. As a check, we calculated the eigenvectors and overlaps for $\chi=\infty, M=75$, eoo symmetric and antisymmetric bases. We found results qualitatively similar to those shown in Figs. 11 and 12, and conclude that the symmetry does not cause deviations from GOE averages.

\section{SUMMARY AND CONCLUSION}

We have presented an investigation of the dependence of the spectral fluctuations and overlap distributions on the classical dynamics, using a schematic three-orbital shell model. Although the model differs qualitatively from previously studied systems, we have found good agreement with the expectations formed by those studies: The spectral averages of several fluctuation measures are equal to GOE ensemble averages when the classical dynamics are chaotic, and equal to Poisson ensemble averages when the classical dynamics are near integrable. We also see the saturation of the $\Delta_{3}$ statistic, and the varia- 


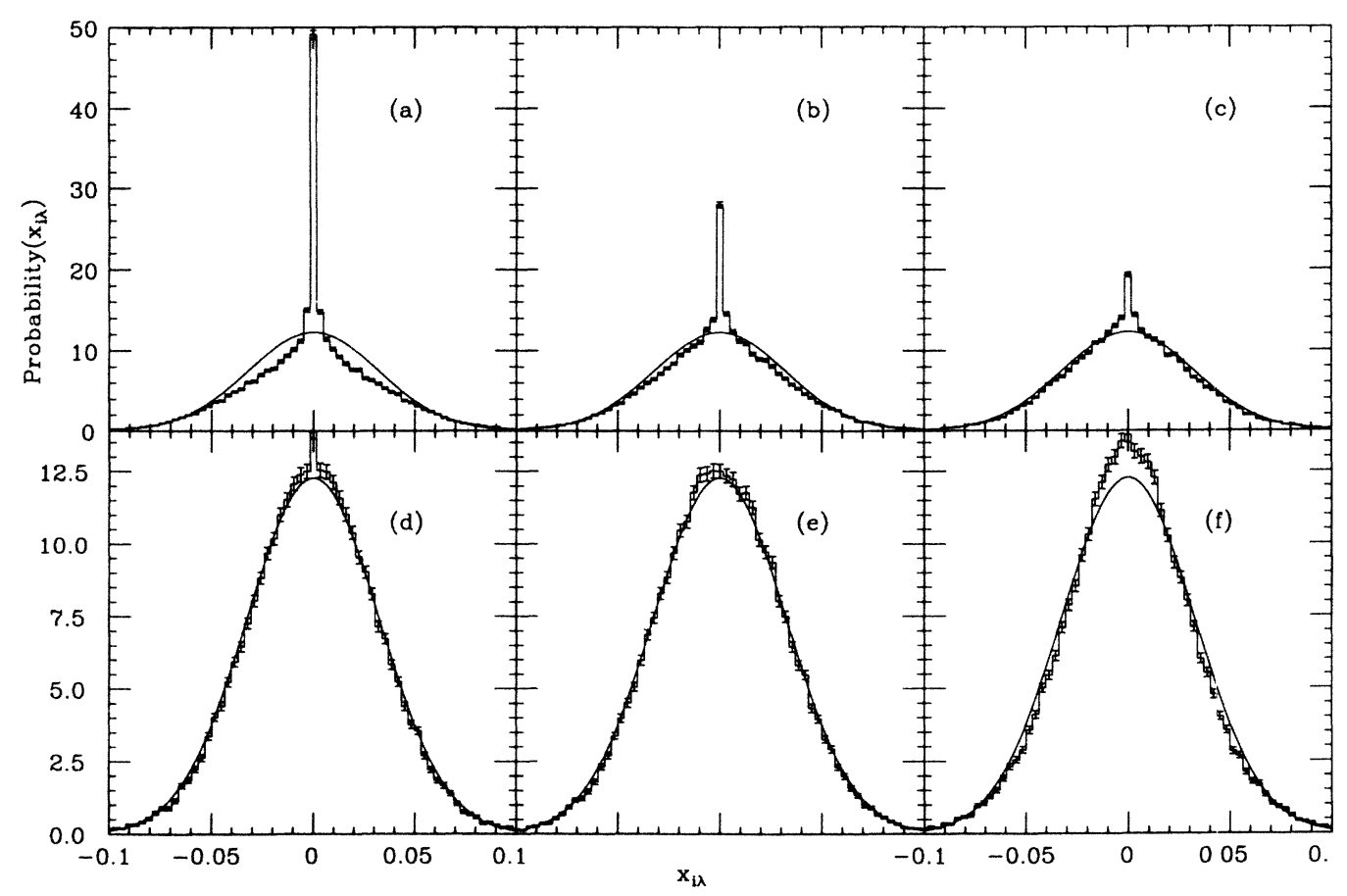

FIG. 12. Histograms of the overlap [Eq. (59)] for the chaotic eigenvectors in the eoe signature class, broken into six energy ranges: panel (a) shows the results for $-25.5<E<-19.0$ (66199 counts); panel (b), the results for $-19.0<E<-14.2$ (65 270 counts); panel (c), for $-14.2<E<-10.2$ (66216 counts); panel (d), for $-10.2<E<-6.6$ (64326 counts); panel (e), for $-6.6<E<-3.3$ (64 323 counts); and panel (f), for $-3.3<E<-0.18$ (67 146 counts). Although the chaotic volume is nearly constant over this interval (Fig. 4), the overlap distribution is clearly not so.

tion of the saturation point with $M$ (number of particles), predicted by the semiclassical arguments of Berry. Finally, while it is well known that discrete symmetries induce Poisson statistics, whatever the classical dynamics, we found that partially conserved discrete symmetries can also. A unique feature of our study was the calculation of overlap (i.e., the projection of an eigenvector onto a basis state) probability distributions, made possible because the basis states are finite in number for the model. The chaotic eigenvectors give nearly the GOE ensemble average (i.e., a Gaussian distribution with a width dependent on the size of the basis). However, there is statistically significant unexplained variation from the GOE average.

\section{ACKNOWLEDGMENTS}

This work, which is based on the thesis by D.C.M., ${ }^{32}$ was supported in part by the National Science Foundation Grant Nos. PHY86-04197 and PHY85-05682. We would also like to acknowledge computer time on the CRAY XMP provided by the San Diego Supercomputing Center.
*Present address: Physics Department, DeMeritt Hall, University of New Hampshire, Durham, NH 03824-3568.

${ }^{\dagger}$ Present address: Institut für Theoretische Physik, Universität zu Köln, Zülpicher Strasse 77, 6000 Köln 41, West Germany.

${ }^{1}$ I. C. Percival, J. Phys. B 6, L229 (1973).

${ }^{2}$ E. J. Heller and M. J. Davis, J. Phys. Chem. 86, 2118 (1982); R. A. Marcus, Faraday Discuss. Chem. Soc. 75, 103 (1983).

${ }^{3}$ O. Bohigas and M.-J. Giannoni, in Mathematical and Computational Methods in Nuclear Physics, edited by J. S. Dehesa, J. M. G. Gomez, and A. Polls (Springer-Verlag, Berlin, 1983).

${ }^{4}$ T. A. Brody, J. Flores, J. B. French, P. A. Mello, A. Pandey, and S. S. M. Wong, Rev. Mod. Phys. 53, 385 (1981).

${ }^{5}$ C. E. Porter, Statistical Theories of Spectra: Fluctuations (Academic, New York, 1965).

${ }^{6}$ R. U. Haq, A. Pandey, and O. Bohigas, Phys. Rev. Lett. 48,
1086 (1982).

${ }^{7}$ M. V. Berry, Ann. Phys. 131, 163 (1981); G. Casati, F. ValzGris, and I. Guarneri, Lett. Nuovo Cimento 28, 279 (1980); S. W. McDonald and A. N. Kaufman, Phys. Rev. Lett. 18, 1189 (1979).

${ }^{8}$ O. Bohigas, M.-J. Giannoni, and C. Schmit, Phys. Rev. Lett. 52, 1 (1984).

${ }^{9}$ T. H. Seligman, J. J. M. Verbaarschot, and M. R. Zirnbauer, Phys. Rev. Lett. 53, 215 (1984); T. H. Seligman, J. J. M. Verbaarschot, and M. R. Zirnbauer, J. Phys. A 18, 2715 (1985).

${ }^{10}$ M. V. Berry and M. Tabor, Proc. R. Soc. London, Ser. A 365, 375 (1977).

${ }^{11}$ M. V. Berry and M. Robnik, J. Phys. A 17, 2413 (1984).

${ }^{12}$ M. V. Berry, Proc. R. Soc. London, Ser. A 400, 229 (1985).

${ }^{13}$ H. Lipkin, N. Meshkov, and A. J. Glick, Nucl. Phys. 62, 188 
(1965); S. Y. Li, A. Klein, and R. M. Dreizler, J. Math. Phys. 11, 975 (1970); G. Holzworth and T. Yukawa, Nucl. Phys. A 219, 125 (1974).

${ }^{14}$ B. Flanders, Ph.D. thesis, California Institute of Technology, 1980, available from University Microfilms International, Ann Arbor, MI 48106; R. D. Williams and S. E. Koonin, Nucl. Phys. A 391, 72 (1982).

${ }^{15}$ W. H. Press, B. P. Flannery, S. A. Teukolsky, and W. T. Vetterling, Numerical Recipes (Cambridge University Press, Cambridge, England, 1986).

16J. P. Blaizot and H. Orland, Phys. Rev. C 24, 1740 (1981).

${ }^{17}$ L. G. Yaffe, Rev. Mod. Phys. 54, 407 (1982).

${ }^{18}$ O. S. van Roosmalen, Ph.D. thesis, University of Groningen, 1982.

${ }^{19}$ K.-K. Kan, Phys. Rev. A 24, 2831 (1981).

${ }^{20} \mathrm{M}$. V. Berry, in Topics in Nonlinear Dynamics, a Tribute to Sir Edward Bullard, edited by S. Journa (AIP, New York, 1978); R. H. G. Helleman, in Fundamental Problems in Statistical Mechanics, edited by E. G. D. Cohen (North-Holland, Amsterdam, 1980), Vol. 5; A. J. Lichtenberg and M. A. Lieberman, Regular and Stochastic Motion (Springer-Verlag, New York, 1983).
${ }^{21}$ G. Benettin, L. Galgani, and J.-M. Strelcyn, Phys. Rev. A 14, 2338 (1976); J.-P. Eckmann and D. Ruelle, Rev. Mod. Phys. 57, 617 (1985); I. Shimada and T. Nagashima, Prog. Theor. Phys. 61, 1605 (1979); A. Wolf, J. B. Swift, H. L. Swinney, and J. A. Vastano, Physica D 16, 285 (1985).

${ }^{22}$ V. I. Arnold, Mathematical Methods of Classical Mechanics (Springer-Verlag, New York, 1978).

${ }^{23}$ M. Henon, Physica D 5, 412 (1982).

${ }^{24}$ E. P. Wigner, SIAM Rev. 9, 1 (1967).

${ }^{25}$ M. Gaudin, Nucl. Phys. 25, 447 (1961).

${ }^{26}$ M. L. Mehta and J. des Cloizeaux, Indian J. Phys. Appl. Math. 3, 329 (1972).

${ }^{27}$ O. Bohigas, R. U. Haq, and A. Pandey, Phys. Rev. Lett. 54, 1645 (1985).

${ }^{28}$ A. Pandey, Ann. Phys. (N.Y.) 119, 170 (1979).

${ }^{29}$ M. C. Gutzwiller, J. Math. Phys. 8, 1979 (1967); 10, 1004 (1969); 11, 1791 (1970); 12, 343 (1971).

${ }^{30}$ R. Balian and C. Bloch, Ann. Phys. 69, 76 (1972).

${ }^{31}$ E. J. Heller, Phys. Rev. Lett. 53, 1515 (1984).

${ }^{32}$ D. C. Meredith, Ph.D. thesis, California Institute of Technology, 1987, available from University Microfilms, Ann Arbor, MI 48106. 

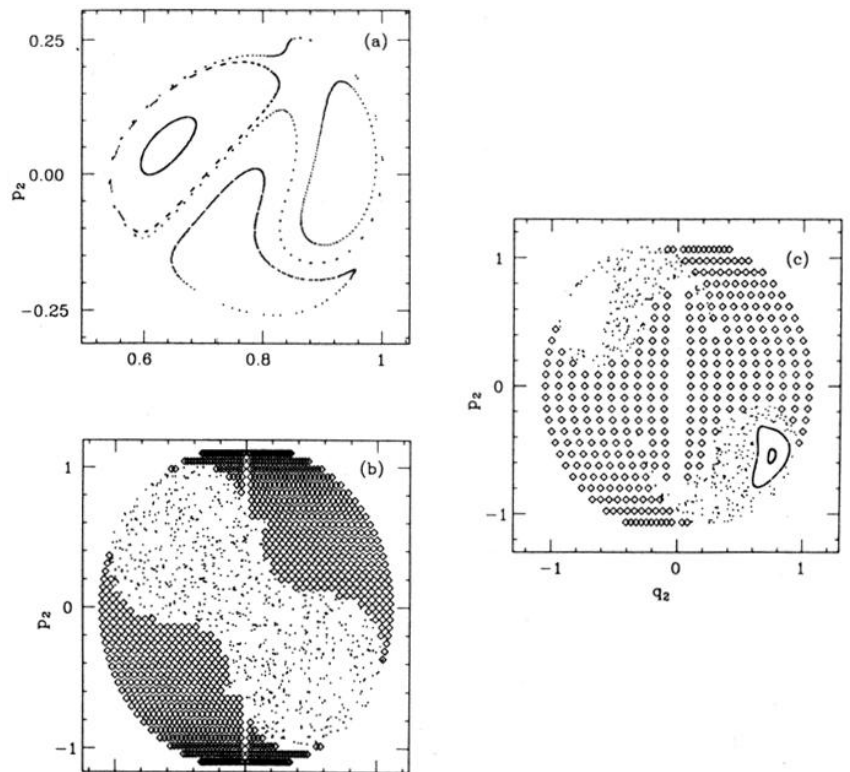

FIG. 2. $\left(q_{2}, p_{2}\right)$ surfaces of section for (a) $E=-30$, (b) $E=-2$, and (c) $E=10$ for the LMG model, with $\chi=100$ and $q_{1}=q_{10}=0.8165$. The diamonds in (b) and (c) are centered on points that are energetically inaccessible. In panel (a) there are five closed curves from five sets of initial conditions; their presence indicates that the dynamics are nearly integrable. Panel (b) shows a sea of points that were generated from one trajectory and that cover the entire available surface; this indicates that the system is ergodic. Panel (c) shows features of the other two, i.e., closed curves and clusters of dots, confirming that the classical dynamics lie between chaotic and integrable. 